\title{
D Antigen Unit
}

National Cancer Institute

\section{Source}

National Cancer Institute. D Antigen Unit. NCI Thesaurus. Code C70501.

A unit of potency of poliovirus vaccine used for poliomyelitis prevention. The unit is poliovirus type-specific. 